Check for updates

Cite this: RSC Adv., 2017, 7, 28350

Received 10th March 2017

Accepted 8th May 2017

DOI: 10.1039/c7ra02937c

rsc.li/rsc-advances

\section{Top-down fabrication of fluorine-doped tin oxide nanopillar substrates for solar water splitting †}

\begin{abstract}
Maureen H. Tang, (D) t $^{\mathrm{a}}$ Pongkarn Chakthranont (D) $t^{\mathrm{b}}$ and Thomas F. Jaramillo (D)*b
Because of its high electronic conductivity, electrochemical stability, and optical transparency, fluorinedoped tin oxide (FTO) is a frequently used substrate for photoelectrochemical water splitting (PEC), dyesensitized solar cells (DSSCs) and other electrocatalytic systems. These applications often require high surface-area substrates, but typical wet-chemical and lithographic approaches to nanostructure this promising material have been limited by the toxic fluorine ion and the resistance of tin oxide to standard chemical etchants. In this work, we develop a novel process to nanostructure commercial FTO by combining nanosphere lithography with argon ion-milling. We show nanostructured FTO with nanopillars of tunable height and diameter. Depositing tungsten oxide with atomic layer deposition on the nanostructured FTO substrate yields a PEC photoanode improvement of $40 \%$ over the baseline FTO substrate. The improvement is ascribed mainly to the increased roughness factor achieved by nanostructuring the substrate.
\end{abstract}

\section{Introduction}

Coating high-surface-area conductive substrates with thin layers of catalytically active materials is a viable strategy to reduce material costs for active materials while improving their activity and stability for a multitude of electrocatalytic and photoelectrocatalytic reactions, including the oxygen evolution reaction (OER). Such an electrode architecture offers more active sites while maintaining short charge transport distances through catalyst materials. The performance of high surface area electrodes relies on good electron transport between the catalytically active materials and the conductive support, as well as the stability of the conductive support at OER conditions. ${ }^{1}$ Although the high surface area of nanostructured electrodes enables larger currents and better performance, it can also amplify corrosion rates. Material stability is therefore of even greater importance. Improved stability of conductive supports at the OER potential is necessary to enable the mass adoption of electrolyzers, photoelectrochemical (PEC) water splitting devices, and anodes for electrochemical water purification. ${ }^{2-4}$ Tin oxide is one of the few support materials that offers stability in an electrochemically corrosive environment and has been investigated as such for polymer electrolyte membrane fuel cells. ${ }^{5}$ As a wide-bandgap semiconductor, $\mathrm{SnO}_{2}$ must be doped

\footnotetext{
${ }^{a}$ Department of Chemical and Biological Engineering, Drexel University, 3141 Chestnut Street, Philadelphia, Pennsylvania 19104, USA

${ }^{b}$ Department of Chemical Engineering, Stanford, University, 443 Via Ortega, California 94305, USA. E-mail: jaramillo@stanford.edu

$\dagger$ Electronic supplementary information (ESI) available. See DOI: $10.1039 / \mathrm{c} 7 \mathrm{ra02937 \textrm {c }}$

$\ddagger$ ORCID: 0000-0003-0037-4814These two authors contribute equally to this work.
}

in order to gain electrical conductivity. Although indium-tin oxide (ITO) is the current state-of-the-art transparent conductive oxide (TCO) for solar cells, it is not stable in an oxidizing environment. ${ }^{6}$ Antimony-doped tin oxide (ATO) has also demonstrated instabilities under extended operation, and the stability is sensitive to synthetic parameters such as annealing time. ${ }^{7,8}$ Fluorine-doped tin oxide (FTO) has so far exhibited the best stability under oxidizing conditions in acidic and basic environments, and has become the substrate of choice for many electrocatalysis and photoelectrocatalysis studies ${ }^{9-14}$ on the basis of its good conductivity, stability, and, for PEC applications, high optical transmissivity.

FTO coatings have been widely adopted to reduce the emissivity of glass windows for improved building efficiency. ${ }^{15}$ Coating processes for commercial FTO have been achieved by chemical-vapor-deposition process (CVD), physical vapor deposition, and sputtering. ${ }^{16}$ Although these methods produces high quality films of low resistance and high optical transmittance, the low roughness of the coatings produced is not ideal for catalysis applications. In contrast, wet-chemical methods such as sol-gel or spray pyrolysis have been used to produce FTO electrodes of high surface area, including nanoparticles, ${ }^{17,18}$ templated inverse opals, ${ }^{19,20}$ and nanowires. ${ }^{21-23}$ Although these structures have demonstrated improved performance over planar samples for DSSC and PEC devices, wet-chemical synthesis routes generally lead to less conductive and less stable TCOs than those synthesized commercially by means of the low-pressure, gas-phase synthesis. $^{24,25}$ Furthermore, wet chemical routes to FTO generally require the use of the highly toxic fluorine ion.

Recognizing the disadvantages to the wet-chemical syntheses of FTO, we have developed a top-down lithographic 
approach to create FTO nanopillars. Tin oxide is resistant to many dry-etch chemistries, and there have been few reports on this subject. ${ }^{26-29}$ Fluorine- and chlorine-based reactive ion etching of $\mathrm{SnO}_{2}$ has been shown to achieve good selectivity with a nickel mask. ${ }^{27}$ Because chlorine was found to damage the tin oxide, other researchers developed etch processes for $\mathrm{SnO}_{2}$ with hydrogen and an organic gas such as ethanol or acetone..$^{\mathbf{2 6} 28}$ Patterning studies of FTO are also limited. One approach is the use of traditional photolithography and $\mathrm{Cl}_{2} / \mathrm{Ar}$ chemistry to etch $150 \mathrm{~nm}$ deep channels. ${ }^{29}$ The conversion efficiency of a DSSC fabricated with the patterned substrate increased from 4.63 to $5.60 \%$. In order to obtain sub-micron lithographic features, nanoimprint lithography and $\mathrm{a}_{2}$ /ethanol reactive ion etch was employed. ${ }^{26}$ The resulting DSSC demonstrated improved light scattering properties and a $2-5 \%$ improvement in internal photon-to-current conversion efficiency. However, the etching selectivity was low, resulting in tapered nanopillars and ridged nanolines.

In this work, we use nanosphere lithography combined with argon ion milling to demonstrate good anisotropy and excellent selectivity. Nanosphere lithography is an inexpensive method for patterning large areas of substrates. ${ }^{30}$ Our approach produces well-ordered nanopillars with tunable heights and diameters, which enhances surface roughness without sacrificing the conductivity and quality of commercial FTO. We further demonstrate the effectiveness of the FTO nanopillars by fabricating ultrathin $\mathrm{WO}_{3}$ photoanodes for PEC water splitting. $\mathrm{WO}_{3}$ is a well-studied photoanode material with various available synthetic methods, including atomic layer deposition (ALD). ${ }^{31,32}$ The ALD process is advantageous for a number of applications because it offers atomically controlled thickness and excellent step coverage. Thus, the surface texturing of the FTO nanopillars can be preserved. ALD of $\mathrm{WO}_{3}$ also utilizes relatively noncorrosive precursors, has a fast growth rate, and produces highly active films without the need for any dopants. ${ }^{33}$ The increased roughness factor of the FTO nanopillars is proven to be advantageous for the ultrathin $\mathrm{WO}_{3}$ photoanode and can be further applied to other photoabsorber and catalyst materials.

\section{Experimental}

\subsection{FTO nanostructuring}

A fabrication process flow diagram of the FTO nanopillars is shown in Fig. 1. FTO-coated glass slides (TEC 8, Hartford Glass Co., $\sim 8 \Omega \mathrm{sq}^{-1}, 10 \mathrm{~mm} \times 15 \mathrm{~mm}$ ) were cleaned sequentially in detergent, acetone, and isopropanol (IPA). A $12 \mathrm{~nm}$ sacrificial layer of aluminum was then evaporated onto the FTO slides (Innotec ES26C E-Beam Evaporator, Innotec). The Al-coated substrates were then rinsed in IPA, dried, and cleaned in oxygen plasma (March Instruments PX-250, $300 \mathrm{~V}$ ) for 45 seconds with $5 \mathrm{sccm} \mathrm{O}_{2}$ to remove organic residue and to improve wetting. $120 \mu \mathrm{L}\left(80 \mu \mathrm{L} \mathrm{cm}{ }^{-2}\right)$ of a mixture of polystyrene nanospheres (Duke Scientific, 5050A, 10\% solids by weight), water, and surfactant ( $400: 1$ by volume of methanol: Triton X-100, Sigma Aldrich) was spin-coated (Brewer Science, CEE 200X) on the substrates to form a monolayer of the hexagonal close packed nanosphere mask. After solution dispersion onto the stationary substrate, the spin-coating program consisted of four stages: (i) wetting the substrate at $0 \mathrm{rpm}$ for $10 \mathrm{~s}$; (ii) spreading the solution at $400 \mathrm{rpm}$ for $5 \mathrm{~s}$ with a ramp rate of $100 \mathrm{rpm} \mathrm{s}^{-1}$; (iii) spinning the substrate at $1600 \mathrm{rpm}$ for $60 \mathrm{~s}$ with a ramp rate of $400 \mathrm{rpm} \mathrm{s}^{-1}$; and finally (iv) removal of excess liquid from the substrate corners at $2000 \mathrm{rpm}$ for $10 \mathrm{~s}$ with a ramp rate of $800 \mathrm{rpm} \mathrm{s}^{-1}$. The mixture compositions for different sphere sizes, modified from literature $^{\mathbf{3 4 , 3 5}}$ are described in Table S1 (ESI $\dagger$ ).

The nanospheres were used as deposited or were reduced in size by exposure to indirect $\mathrm{O}_{2}$ plasma for $60 \mathrm{~s}$ (260 $\mathrm{nm}$ sphere (a) Evaporate Al on FTO
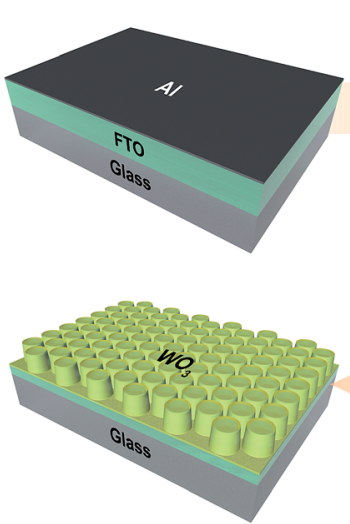

(f) Deposit $\mathrm{WO}_{3}$ by ALD (b) Spin coat a monolayer of polystyrene spheres
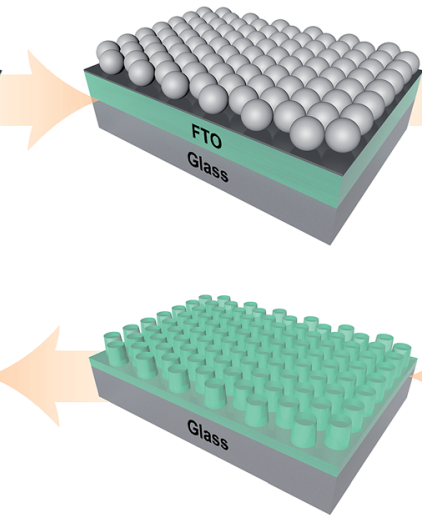

(e) Remove Al and the sphere residue (c) Dry etch with $\mathrm{O}_{2}$ plasma to reduce the sphere diameter
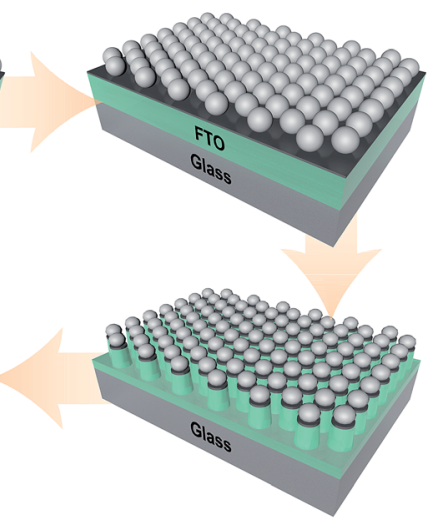

(d) Ar ion mill to transfer the pattern

Fig. 1 Fabrication process of the $\mathrm{WO}_{3}$ coated FTO nanopillars for the photoelectrochemical water oxidation reaction. (a) A $12 \mathrm{~nm}$ sacrificial Al layer is deposited on commercial FTO-coated glass by physical vapor deposition. (b) A monolayer of polystyrene nanospheres (500 or $260 \mathrm{~nm}$ in diameter) is formed on the Al layer by spin coating. (c) The nanosphere mask is further reduced in size by dry etching with $\mathrm{O}_{2}$ plasma. (d) FTO columns are formed by anisotropic etching of the FTO substrate with argon ion milling. (e) The mask is then lifted off in $10 \mathrm{M} \mathrm{NaOH}$ and cleaned in piranha solution. (f) Lastly, crystalline $\mathrm{WO}_{3}$ is deposited by plasma-assisted atomic layer deposition. 
diameter) or $180 \mathrm{~s}$ (500 $\mathrm{nm}$ sphere diameter), then mounted on a carrier wafer with Kapton tape and ion-milled for 11 minutes in an Intlvac Nanoquest Research Ion Beam Mill System at $400 \mathrm{~V}, 50 \mathrm{~mA}, 15 \mathrm{sccm}$ argon, and 90 degree stage angle. After ion-milling, the polystyrene sphere residues and sacrificial $\mathrm{Al}$ were removed by sonication in $10 \mathrm{M} \mathrm{NaOH}$. The substrates were then rinsed in water and cleaned a final time in a piranha solution $\left(3: 1 \mathrm{H}_{2} \mathrm{SO}_{4}: \mathrm{H}_{2} \mathrm{O}_{2}\right)$.

\section{$2.2 \mathrm{WO}_{3}$ photoabsorber synthesis}

Prior to $\mathrm{WO}_{3}$ synthesis, the native FTO and FTO nanopillars were cleaned in $\mathrm{O}_{2}$ plasma for 5 minutes to remove any adventitious organics (Drytek Model DRIE-100 Plasma Etcher). $5 \AA$ of $\mathrm{Al}_{2} \mathrm{O}_{3}$ was then applied onto the FTO substrates as an adhesion layer ( 5 cycles of atomic layer deposited $\mathrm{Al}_{2} \mathrm{O}_{3}$ at 200 ${ }^{\circ} \mathrm{C}$ ). An ultrathin $\mathrm{WO}_{3}$ film was synthesized by atomic layer deposition (ALD) in a Fiji F202 system (Cambridge Nanotech) at $350{ }^{\circ} \mathrm{C}$. An ALD cycle of $\mathrm{WO}_{3}$ consisted of pulsing bis(tertbutylimino)-bis(dimethylamino)tungsten(vi) (BTBMW, SigmaAldrich) at $85{ }^{\circ} \mathrm{C}$ followed by $\mathrm{O}_{2}$ plasma for $20 \mathrm{~s}(300 \mathrm{~W})$. The pulse time of BTBMW was varied from $0.1-3 \mathrm{~s}$ in order to verify the surface absorption window of the $\mathrm{W}$ precursor. The reactor chamber was purged for $20 \mathrm{~s}$ with $30 \mathrm{sccm}$ of argon between each precursor pulse. The thickness of ALD tin oxide films grown on silicon wafers was measured by a spectroscopic ellipsometer (Woollam M2000). $20 \mathrm{~nm}$ of $\mathrm{WO}_{3}$ was deposited on various FTO substrates and then calcined in a tube furnace at $550{ }^{\circ} \mathrm{C}$ in air for 1 hour.

\subsection{Characterization}

The structures and morphologies of the bare FTO nanopillars and the $\mathrm{WO}_{3}$ coated FTO electrodes were imaged using scanning electron microscopy (SEM) (FEI Magellan XHR microscope operated with a beam voltage of $5.0 \mathrm{kV}$ and current of $25 \mathrm{pA}$ ). The surface roughness was characterized by atomic force microscopy (AFM) using a Park XE-70 in non-contact mode with Si tip and $37 \mathrm{~N} \mathrm{~m}^{-1}$ force. The average roughness factor (RF) value, which is a ratio between a true surface area and a geometric surface area, was calculated using the XEI data analysis program. The sheet resistance was measured by a round-tip 4-point probe with $1.0 \mathrm{~mm}$ distance between each probe tip. The crystallinity of the $\mathrm{WO}_{3}$ was studied with ambient temperature grazing incidence X-ray diffraction (GIXRD) (Phillips PANanalytical X'Pert Pro) using $\mathrm{Cu} \mathrm{K} \alpha$ radiation $(\lambda=$ $1.54056 \AA$ ). The incidence angle $(\omega)$ was $0.75^{\circ}$ and the attenuator slit was $1 / 8^{\circ}$. X-ray photoelectron spectroscopy (XPS) was performed using a Phi VersaProbe spectrometer with an $\mathrm{Al} \mathrm{K \alpha}$ source. The absorbance was collected inside a custom-fitted integrating sphere ( 8 " AdaptaSphere, Labsphere Inc.) with illumination from a $1000 \mathrm{~W}$ Xe lamp (Newport-Oriel). The ideal current density was calculated from the absorbance above the band gap, subtracted by the blank substrate absorbance.

\subsection{Photoelectrochemical testing}

All electrochemistry was performed using a BioLogic VSP potentiostat with the EC-Lab software. Capacitance measurements were performed as previously described.$^{36}$ Cyclic voltammetry was performed in $0.5 \mathrm{M} \mathrm{H}_{2} \mathrm{SO}_{4}$ with a platinum counter electrode and $\mathrm{Ag} / \mathrm{AgCl}$ reference electrode. The electrode potential was swept from 0.05 to $0.15 \mathrm{~V} v s$. reference at scan rates from 50 to $1000 \mathrm{mV} \mathrm{s}^{-1}$, and the capacitive current was obtained by averaging the anodic and cathodic current at $0.10 \mathrm{~V}$ vs. Ag/AgCl.

A photoelectrode was prepared by contacting a $\mathrm{Cu}$ wire to the FTO substrate with conductive paint (Electrodag 502, Ted Pella Inc.) and encasing the contact in insulating epoxy (Loctite Hysol, 9462). The geometric surface areas were measured from photographs with ImageJ software. Photoelectrochemical testing was done in a three-electrode electrochemical setup with a Pt coil counter electrode and a $\mathrm{Ag} / \mathrm{AgCl}(4 \mathrm{M} \mathrm{KCl})$ reference electrode. The electrode was fully submerged in argon-saturated $0.1 \mathrm{M} \mathrm{H}_{2} \mathrm{SO}_{4}$ electrolyte ( $\mathrm{pH} 1$ ) in a heart-shaped glass cell (pine) with a flat front-facing window for illumination. The cyclic voltammetry measurements were performed at a $25 \mathrm{mV} \mathrm{s}^{-1}$ scan rate under $2 \mathrm{~s}$ chopped illumination from the front side (through the $\mathrm{WO}_{3}$ layer). All the photoelectrochemistry data in this work are reported at the potential $v s$. RHE scale without iR compensation.

A $1000 \mathrm{~W}$ xenon lamp (Newport-Oriel) provided concentrated broadband illumination. The power density of the utilizable photons (wavelength from 280 to $460 \mathrm{~nm}$ ) was $15.52 \mathrm{~mW} \mathrm{~cm}^{-2}$, which matches the power density of AM 1.5 G spectrum (ASTM G-173-03) for that same wavelength range. The power was measured by a spectrometer (Jaz, Ocean Optics Inc.).

\section{Results and discussion}

\subsection{Formation of FTO nanopillars}

SEM images of the FTO nanopillars at various stages of processing are shown Fig. 2. Fig. 2a and e show the excellent ordering obtained by the spin-coating process. Comparing (a) and (e) reveals that fewer defects are present for the $500 \mathrm{~nm}$ spheres, likely because the larger spheres are less influenced by the native roughness of the FTO substrate. Fig. $2 \mathrm{~b}$ and $\mathrm{f}$ show the reduction in sphere size upon exposure to the $\mathrm{O}_{2}$ plasma. The reduction of the sphere size is a critical step for the formation of the pillar structure, as will be further discussed below.

Fig. 2c and $\mathrm{g}$ demonstrate the anisotropy and selectivity of the ion milling process. Despite etching hundreds of $\mathrm{nm}$ deep into the FTO, the feature diameter in Fig. 2c only decreased from 430 to approximately $420 \mathrm{~nm}$. This high Ar-milling selectivity is unique to polystyrene nanospheres and is not observed when using silica or alumina nanospheres as masks. This is because, upon exposure to the argon ions, the polymer becomes highly cross-linked and resistive to further etching, while silica and alumina masks are etched by argon ions at roughly the same rate as the underlying FTO.

Fig. $2 \mathrm{~d}$ and $\mathrm{h}$ show the FTO nanopillars after a mask lift-off process. In the absence of the sacrificial $\mathrm{Al}$ layer, we were unable to remove the sphere residues using organic solvents, aggressive oxygen plasmas, calcination, or piranha etchants. However, the deposition of $12 \mathrm{~nm}$ of aluminum before spin-coating 

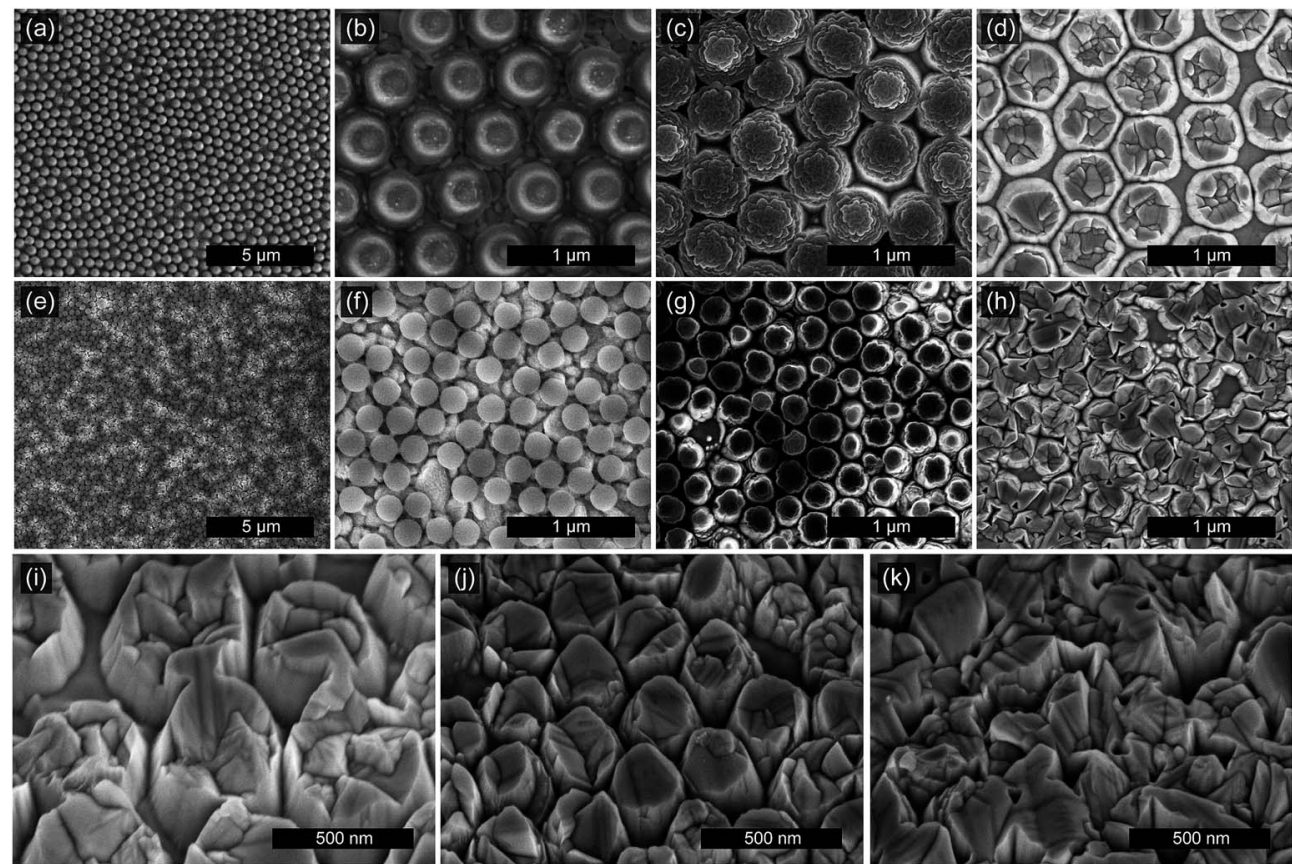

Fig. 2 SEM images of the FTO nanopillars at various processing stages using $500 \mathrm{~nm}$ spheres (first row) and $260 \mathrm{~nm}$ spheres (second row) as patterning masks. (a and e) A monolayer of nanospheres was deposited on FTO substrate by spin-coating. (b and f) The spheres were shrunk in $\mathrm{O}_{2}$ plasma. ( $c$ and g) The FTO substrates were milled by argon ion beam. ( $d$ and h) After mask removal, nanopillar structures were achieved. $30^{\circ}$ tilt and higher magnification SEM images of nanostructured FTO substrates (third row). Reducing the diameters of the (i) $500 \mathrm{~nm}$ or (j) $260 \mathrm{~nm}$ spheres in $\mathrm{O}_{2}$ plasma prior to milling was necessary to form nanopillars, otherwise only narrow pits are formed between fused spheres, as shown in $(k)$ where no sphere reduction was employed.

provides a sacrificial layer that is soluble in strong base. Dissolving the aluminum also lifts off the sphere residues, yielding a clean surface of FTO nanopillars.

Fig. 2i-k show additional SEM images of three samples at $30^{\circ}$ tilt and at higher magnification, revealing the importance of reducing sphere diameter in the $\mathrm{O}_{2}$ plasma prior to ion milling. Fig. $2 \mathrm{i}$ and $\mathrm{j}$ show a different view of the nanopillars formed from $500 \mathrm{~nm}$ and $260 \mathrm{~nm}$ spheres respectively, consistent with images shown in Fig. 2a-h. If the sphere diameter is not reduced, necking between the spheres limits ion milling to narrow channels, and distinct nanopillars are not formed, as shown in Fig. 2k. We expect that further decreasing the sphere diameter could provide a method to separately tune the nanopillar diameter and spacing, motivating future work. Fig. $2 \mathrm{i}$ and $\mathrm{j}$ show slight tapering of the nanopillars. This is partly caused by imperfect anisotropy during the milling, but also because initially, the polymer nanospheres have not yet formed the crosslinked network, and the perimeter of the sphere is also milled away. Because the smaller spheres have a higher surfacearea-to-volume ratio, this preliminary milling at the edge is relatively more significant and results in lower overall milling selectivity and more tapered nanopillars.

\subsection{Roughness factors and optical properties of FTO nanopillars}

For PEC, DSSC, and catalysis applications, higher roughness factors are often desired. The roughness factors of the nanostructured FTO substrates were measured using AFM and double-layer capacitance. AFM images are shown in Fig. 3a-c and a representative double-layer capacitance chart is shown in Fig. $3 \mathrm{e}$ and $\mathrm{f}$. The resulting roughness factors, normalized to a native FTO, are summarized in Table 1. Good agreement is observed between the two methods of surface area measurement. Also shown in Table 1 is the sheet resistance of different FTO substrates, which only slightly increases with the roughness factor. Although the observed relative roughness factors are relatively small (a factor of 1.4X over native FTO), the etch depth in Fig. 3d is limited not by the selectivity of the mask, but by the thickness of the native FTO. A thicker FTO layer starting material would permit deeper milling and thus a higher roughness factor. FTO that was nanostructured without reducing the sphere diameter exhibits a lower surface area, consistent with the SEM images of interconnected spheres.

For PEC applications, substrates must also be optically transparent. UV-vis absorbance of the nanostructured FTO, as shown in Fig. 4, does not result in significant increases in absorption after the texturing process.

\section{3 $\mathrm{WO}_{3} \mathrm{ALD}$ characterizations}

Before coating the FTO nanopillars, the ALD $\mathrm{WO}_{3}$ process was investigated on Si planar substrates to determine the surface saturation regime and to verify a linear growth rate. A BTBMW pulse time of $2 \mathrm{~s}$ was chosen to ensure high coverage of the $\mathrm{W}$ precursor on the substrate surface, resulting in the $\mathrm{WO}_{3}$ growth 

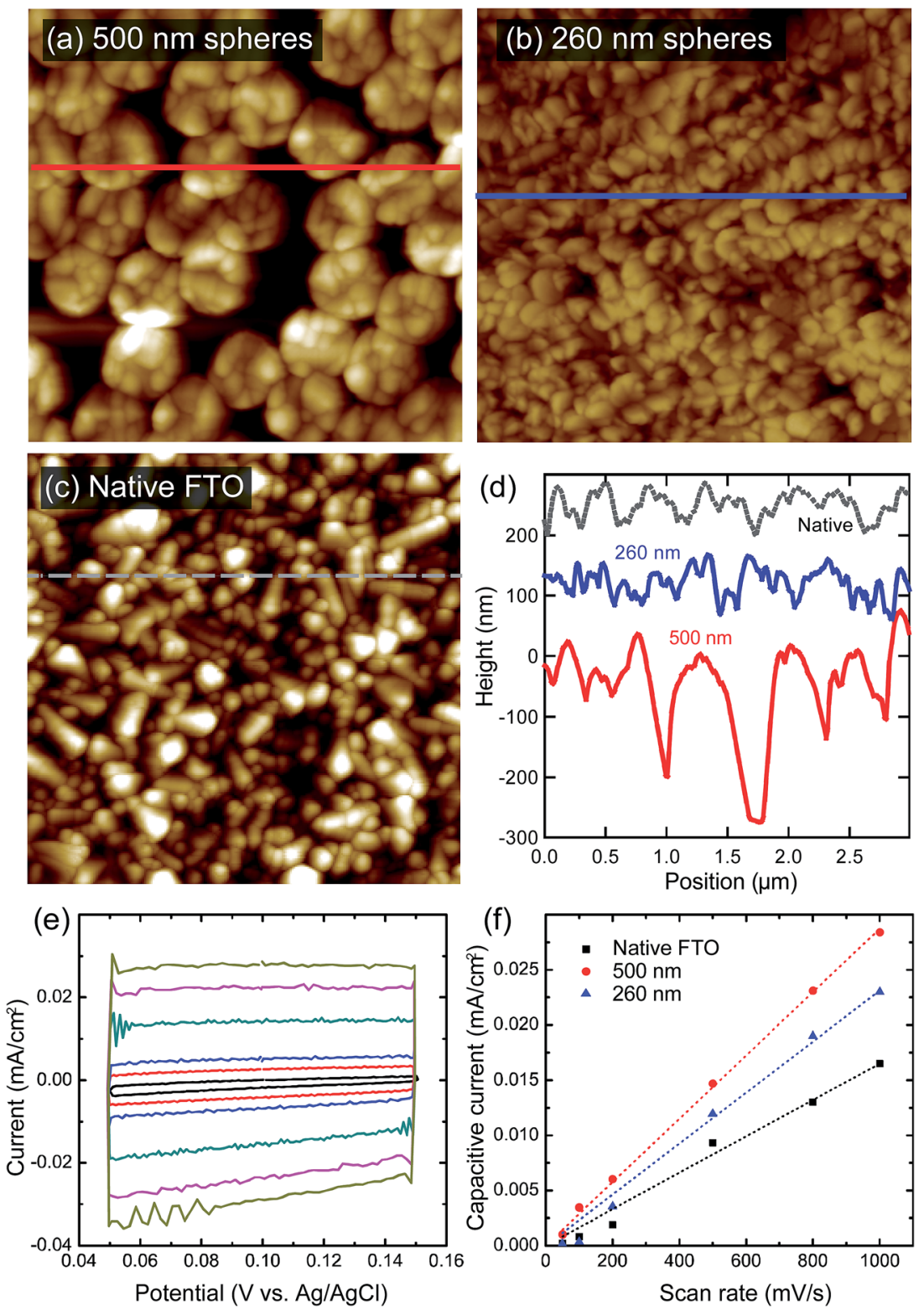

Fig. 3 AFM images of the FTO nanopillars: (a) FTO nanopillars formed by $500 \mathrm{~nm}$-sphere mask, (b) $260 \mathrm{~nm}$-sphere mask, and (c) a native FTO. (d) The height measured by AFM conveys the roughness factor of each substrate. (e) Double layer capacitance current densities of 500 nm FTO nanopillars measured by cyclic voltammetry at different scan rates from 50 to $1000 \mathrm{mV} \mathrm{s}^{-1}$. (f) The capacitance current densities extracted from the $\mathrm{CVs}$ at $0.10 \mathrm{~V} v \mathrm{~s}$. $\mathrm{Ag} / \mathrm{AgCl}$ are linear functions of the scan rate.

Table 1 Normalized roughness factor to a native FTO measured by AFM and capacitive current, and sheet resistance of different substrates

\begin{tabular}{llll}
\hline & \multicolumn{2}{l}{ Normalized RF } & \\
\cline { 2 - 3 } Sample & AFM & Capacitive current $^{*}$ & Sheet resistance $\left(\Omega\right.$ sq $\left.^{-1}\right)$ \\
\hline $\begin{array}{l}\text { Baseline FTO } \\
500 \text { nm nanopillars, no sphere }\end{array}$ & 1 & 1 & 7.2 \\
$\begin{array}{l}\text { reduction } \\
500 \text { nm nanopillars, sphere reduction }\end{array}$ & 1.2 & 1.3 & 8.2 \\
$\begin{array}{l}260 \text { nm nanopillars, no sphere } \\
\text { reduction }\end{array}$ & 1.2 & 1.4 & 10.0 \\
260 nm nanopillars, sphere reduction & 1.0 & 1.3 & 9.6 \\
\end{tabular}




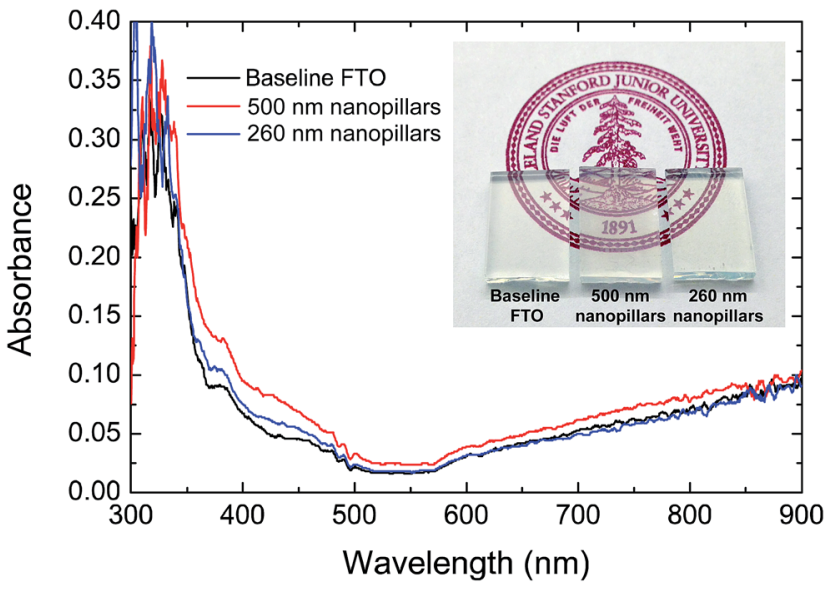

Fig. 4 UV-vis absorbance of native FTO substrate and FTO nanopillars.

(a)

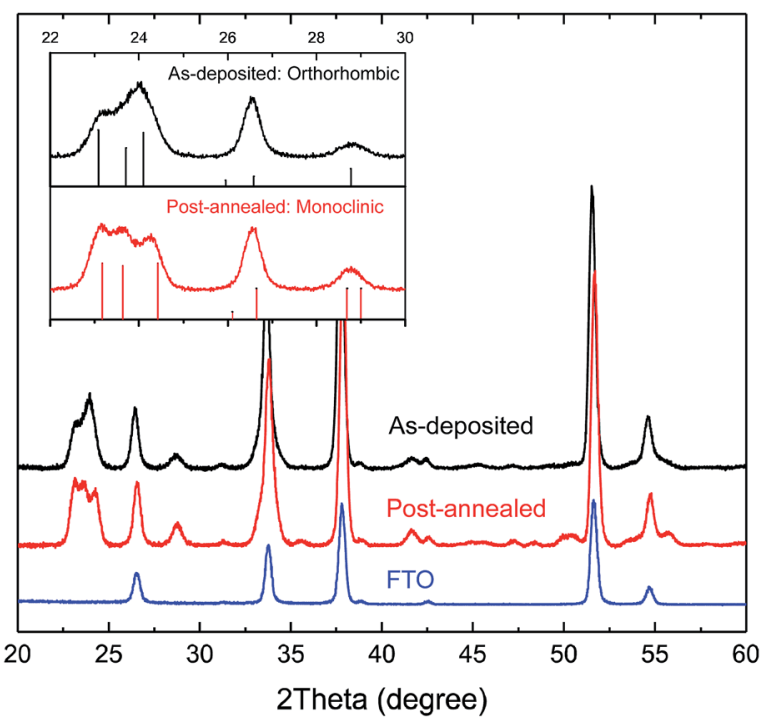

(b)

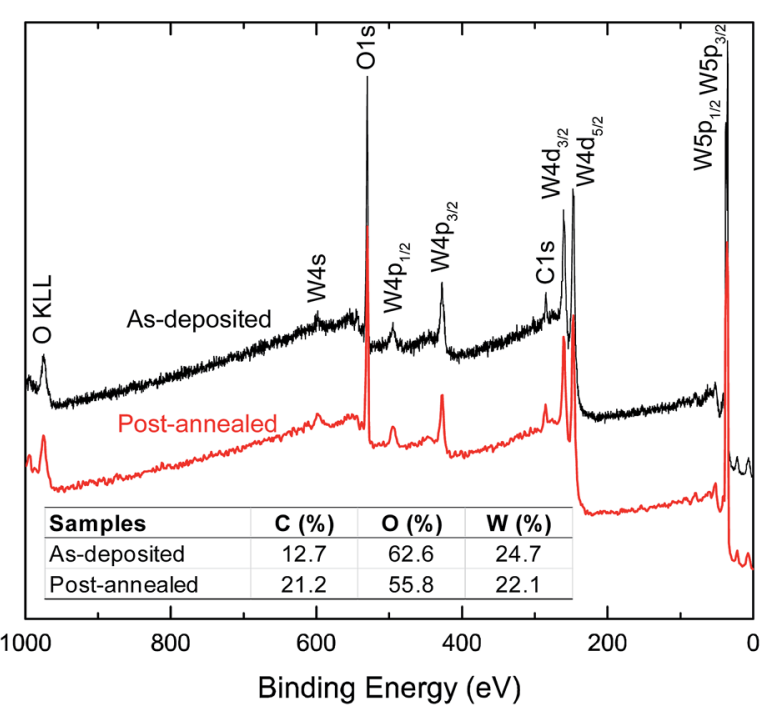

Fig. 5 GIXRD and XPS spectra of ALD WO $W_{3}$. (a) GIXRD patterns and (b) XPS spectra of the as-deposited and post-annealed ALD $\mathrm{WO}_{3}$ show that the films consist of crystalline $\mathrm{WO}_{3}$. rate of $0.9 \AA$ A per cycle (Fig. S1†). XRD patterns show that the asdeposited ALD $\mathrm{WO}_{3}$ film exhibits an orthorhombic structure, and the post-annealed film is converted to monoclinic $\mathrm{WO}_{3}$ (Fig. 5a), similar to active $\mathrm{WO}_{3}$ reported in literature. ${ }^{33}$ XPS spectra confirm that the surface of the films consist of $\mathrm{WO}_{3}$ with a carbon content typical of adventitious sources (Fig. 5b).

\subsection{PEC performance}

Fig. 6a shows the PEC performance of $20 \mathrm{~nm}$ thick $\mathrm{WO}_{3}$ photoanodes. All the $\mathrm{WO}_{3}$-coated FTO nanopillar samples exhibit higher photocurrent density than the equivalent $\mathrm{WO}_{3}$ coated onto a native FTO electrode. The highest photocurrent density was achieved with nanopillar substrates fabricated with $500 \mathrm{~nm}$ diameter nanospheres reduced in oxygen plasma for $180 \mathrm{~s}$, a $40 \%$ improvement over the use of a native FTO substrate.

Fig. 6b shows that the current density (at $2 \mathrm{~V}$ vs. RHE) increases linearly with the ideal current density calculated from absorption properties, which indicates direct use of the
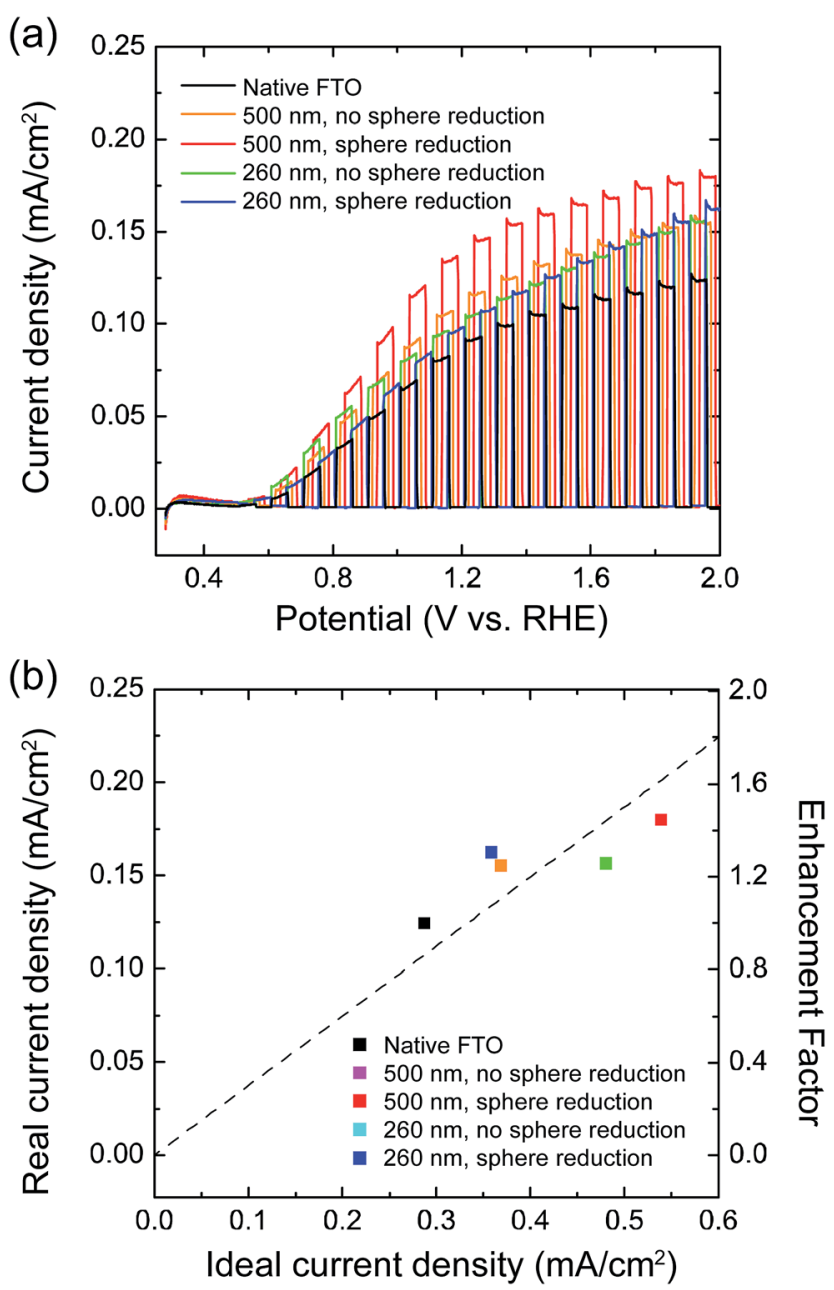

Fig. 6 Photoelectrochemical performances ALD $\mathrm{WO}_{3}$ on FTO nanopillars. (a) Photoelectrochemical performance of $20 \mathrm{~nm} \mathrm{WO}$ photoanodes on various FTO substrates under chopped 1-sun illumination. (b) The real current density at $2 \mathrm{~V} v \mathrm{vs}$. RHE and the photocurrent enhancement factor $\left(\mathrm{WO}_{3}\right.$ on native FTO =1) vs. the ideal current density calculated from the absorption. 
increased material deposited with no apparent loss from charge transport. Because the current density enhancement factors on nanostructured FTO substrates are similar to their roughness factors reported in Table 1, we can conclude that the increased roughness is the primary cause for this performance enhancement. The proportionate relationship of roughness factor and current enhancement factor implies that the interfacial recombination is not a performance limiting factor because of the negligible change in the substrate quality and the relatively low roughness factor. To improve the PEC performance even further, a thicker native FTO could be used to for higher aspect ratio structures with greater roughness factors.

\section{Conclusion}

FTO nanopillars were successfully fabricated using a top-down fabrication process consisting of nanosphere lithography and argon ion milling. By varying the sphere size, the sphere reduction time, and the milling duration, the profile and the roughness factor of the FTO nanopillars can be tuned. These FTO nanopillars exhibit the desired properties of excellent ordering, low optical absorption, and low sheet resistivity. The nanopillars were used as a transparent conductive substrate for an ultrathin $\mathrm{WO}_{3}$ PEC electrode synthesized by plasma-assisted ALD. Although the enhanced roughness factor of the FTO nanopillars was limited by the thickness of the starting film of FTO, it was proven to be beneficial for the $\mathrm{WO}_{3}$ photoanode; the enhancement in photocurrent was found to be equal to the increase in roughness factor of the substrate. This result demonstrates that the FTO nanopillars can increase the optical density of the absorber material while maintaining short charge transport distances. Our top-down approach to nanostructuring enhances the roughness factor on commercial FTO without sacrificing the high quality of the substrate, and can be readily generalized for other commercially available conductive substrates and other photoabsorber materials for a variety of applications.

\section{Acknowledgements}

This paper presents results from an NSF project (award number CBET-1433442) competitively-selected under the solicitation "NSF 14-15: NSF/DOE Partnership on Advanced Frontiers in Renewable Hydrogen Fuel Production via Solar Water Splitting Technologies", which was co-sponsored by the National Science Foundation, Division of Chemical, Bioengineering, Environmental, and Transport Systems (CBET), and the U.S. Department of Energy, Office of Energy Efficiency and Renewable Energy, Fuel Cell Technologies Office. The authors would like to thank Dr Blaise Pinaud, Ieva Narkeviciute, Dr Michelle Rincon, and Dr J Provine for helpful discussions.

\section{References}

1 J. Moir, N. Soheilnia, P. O'Brien, A. Jelle, C. M. Grozea, D. Faulkner, M. G. Helander and G. A. Ozin, ACS Nano, 2013, 7, 4261-4274.
2 Z. Zhang, J. Liu, J. Gu, L. Su and L. Cheng, Energy Environ. Sci., 2014, 7, 2535-2558.

3 M. G. Walter, E. L. Warren, J. R. McKone, S. W. Boettcher, Q. Mi, E. A. Santori and N. S. Lewis, Chem. Rev., 2010, 110, 6446-6473.

4 B. P. Chaplin, Environ. Sci.: Processes Impacts, 2014, 16, 11821203.

5 Y. Shao, J. Liu, Y. Wang and Y. Lin, J. Mater. Chem., 2008, 19, 46-59.

6 J. D. Benck, B. A. Pinaud, Y. Gorlin and T. F. Jaramillo, PLoS One, 2014, 9, e107942.

7 Q. Peng, B. Kalanyan, P. G. Hoertz, A. Miller, D. H. Kim, K. Hanson, L. Alibabaei, J. Liu, T. J. Meyer and G. N. Parsons, Nano Lett., 2013, 13, 1481-1488.

8 E. Fabbri, A. Rabis, R. Kotz and T. J. Schmidt, Phys. Chem. Chem. Phys., 2014, 16, 13672-13681.

9 Y. Liang, T. Tsubota, L. P. A. Mooij and R. van de Krol, J. Phys. Chem. C, 2011, 115, 17594-17598.

10 Y. J. Hwang, C. Hahn, B. Liu and P. Yang, ACS Nano, 2012, 6, 5060-5069.

11 R. Brimblecombe, A. Koo, G. C. Dismukes, G. F. Swiegers and L. Spiccia, J. Am. Chem. Soc., 2010, 132, 2892-2894.

12 G. K. Mor, H. E. Prakasam, O. K. Varghese, K. Shankar and C. A. Grimes, Nano Lett., 2007, 7, 2356-2364.

13 T. Hoshikawa, M. Yamada, R. Kikuchi and K. Eguchi, J. Electrochem. Soc., 2005, 152, E68-E73.

14 J. J. Concepcion, J. W. Jurss, M. K. Brennaman, P. G. Hoertz, A. O. T. Patrocinio, N. Y. Murakami Iha, J. L. Templeton and T. J. Meyer, Acc. Chem. Res., 2009, 42, 1954-1965.

15 Pilkington Group Limited, Energy Saving Glass - Warm Coatings, http:/www.pilkington.com/pilkington-information/ about+pilkington/education/glass+in+buildings/coatings.htm, accessed Jan 18, 2017.

16 B. Stjerna, E. Olsson and C. Granqvist, J. Appl. Phys., 1994, 76, 3797-3817.

17 T. P. Chou, Q. Zhang, B. Russo and G. Cao, J. Nanophotonics, 2008, 2, 023511.

18 Z. Yang, S. Gao, T. Li, F.-Q. Liu, Y. Ren and T. Xu, ACS Appl. Mater. Interfaces, 2012, 4, 4419-4427.

19 J. Xiao, L. Yang, Y. Luo, D. Li and Q. Meng, J. Mater. Chem. C, 2013, 1, 5450-5453.

20 Z. Yang, S. Gao, W. Li, V. Vlasko-Vlasov, U. Welp, W.-K. Kwok and T. Xu, ACS Appl. Mater. Interfaces, 2011, 3, 1101-1108.

21 B. Russo and G. Cao, Appl. Phys. A, 2008, 90, 311-315.

22 K. Murakami, A. Bandara, M. Okuya, M. Shimomura and R. M. G. Rajapakse, Synthesis and characterization of onedimensional nanostructured fluorine-doped tin dioxide thin films, Proc. SPIE 9929, Nanostructured Thin Films IX, 99290X, 2016.

23 A. Bandara, K. Murakami, R. M. G. Rajapakse, P. V. V. Jayaweera, M. Shimomura, H. M. N. Bandara, D. Liyanage and E. V. A. Premalal, Thin Solid Films, 2017, 621, 229-239.

24 T. Minami, Semicond. Sci. Technol., 2005, 20, S35.

25 H. Bisht, H.-T. Eun, A. Mehrtens and M. Aegerter, Thin Solid Films, 1999, 351, 109-114. 
26 F. Wang, N. K. Subbaiyan, Q. Wang, C. Rochford, G. Xu, R. Lu, A. Elliot, F. D'Souza, R. Hui and J. Wu, ACS Appl. Mater. Interfaces, 2012, 4, 1565-1572.

27 J. C. Park, J. K. Kim, T. G. Kim, D. W. Lee, H. Cho, H. S. Kim, S. J. Yoon and Y.-G. Jung, Int. J. Mod. Phys. B, 2011, 25, 42374240.

28 T. Minami, T. Miyata, A. Iwamoto, S. Takata and H. Nanto, Jpn. J. Appl. Phys., Part 2, 1988, 27, L1753.

29 S. M. Kong, Y. Xiao, K. H. Kim, W. I. Lee and C. W. Chung, Thin Solid Films, 2011, 519, 3173-3176.

30 C. L. Haynes and R. P. Van Duyne, J. Phys. Chem. B, 2001, 105, 5599-5611.
31 X. Liu, F. Wang and Q. Wang, Phys. Chem. Chem. Phys., 2012, 14, 7894-7911.

32 J. Malm, T. Sajavaara and M. Karppinen, Chem. Vap. Deposition, 2012, 18, 245-248.

33 R. Liu, Y. Lin, L. Y. Chou, S. W. Sheehan, W. He, F. Zhang, H. J. Hou and D. Wang, Angew. Chem., 2011, 123, 519-522.

34 C. L. Cheung, R. Nikolić, C. Reinhardt and T. Wang, Nanotechnology, 2006, 17, 1339.

35 B. A. Pinaud, Fundamental studies for the design of tantalum nitride photoanodes for solar water splitting, Stanford University, 2013.

36 A. J. Forman, Z. Chen, P. Chakthranont and T. F. Jaramillo, Chem. Mater., 2014, 26, 958-964. 